\title{
In Search for Effective and Safe Drugs against SARS-CoV-2: Part I] Simulated interactions between selected nutraceuticals, ACE2 enzyme and S Protein simple peptide sequences
}

\author{
M. S. A. Abdel-Mottaleb ${ }^{1}$ and Yousra Abdel-Mottaleb ${ }^{2}$ \\ ${ }^{1}$ Department of Chemistry, Faculty of Science, Ain Shams University (ASU), Abbassia 11566, \\ Cairo, Egypt \\ ${ }^{2}$ Department of Pharmacology, Toxicology and Biochemistry, Faculty of Pharmaceutical \\ Sciences and Pharmaceutical Industries, Future University in Egypt (FUE), New Cairo 11835, \\ Egypt
}

Corresponding author: m.s.abdelmottaleb@sci.asu.edu.eg

1https://orcid.org/0000-0002-4437-7040

${ }^{2}$ https://orcid.org/0000-0002-4327-4920

\begin{abstract}
:
Coronavirus disease (COVID-19) remains a world pandemic with little treatment options. Nature has provided a plethora of compounds that may offer potential protection and/or treatment choices. Earlier studies have shown a pivotal role of Angiotensin converting enzyme 2 (ACE2) in the pathogenesis of COVID-19. In this context, seven natural compounds were selected and their binding to specific peptide sequences of the coronavirus S-protein: ACE2 interface-drug binding adduct were calculated. Further to the natural drugs, we also similarly examined four well-known antiviral drugs. Moreover, the binding-interface of the isolated coronavirus S-protein and the isolated ACE2 receptor were also individually explored. The identified drug molecules positioned itself achieving geometries of minimum energy resulting in limiting viral recognition of host cells or to disturb host-virus interactions. The frontier orbitals (HOMO-LUMO) play crucial role in the binding interactions of the studied molecules. Most of the drugs act as electron sink whereas the $S$ protein behaves as nucleophile. The results reported pave the way for the identification of smalldrug molecule of natural origin with potentially tolerable side effects that can offer protection and/or treatment against coronavirus S-protein COVID-19. Experimental validation is of urgent demand.
\end{abstract}

Keywords: Viral S protein, ACE2, Antiviral Drugs, Frontier Orbitals, Binding Energy. 


\section{1- Introduction}

The Coronavirus disease 2019(COVID-19) is raging throughout the world since December 2019. The treatment options remain unsatisfactory and scarce. Taking in consideration the urgency of the current worldwide situation, repurposing preexisting drugs and nutraceuticals seems like a reasonable option [1]. For instance, drugs such as the antiviral favipiravir, which selectively inhibits RNA polymerase and prevents replication of the viral genome [2], the antimalarial hydroxychloroquine, which affects the binding of ACE2 and SARS-CoV2 spike protein, the antiparasitic ivermectin with nuclear transport inhibitory activity [3] and finally the anthelmintic niclosamide [4] have received attention as potential therapeutic agents tackling the virus through different mechanisms. Additionally, several well-known natural agents [5-8] such as curcumin, menthol, eriodictyol, thymoquinone, resveratrol, quercetin and raspberry ketone could potentially offer antiviral effects [9-15]. The combination of two to multiple agents could offer endless therapeutic options.

Earlier studies have proposed a few mechanisms of interaction of SARS-CoV with the host cells:

1. Spike glycoprotein with ACE2. The spike glycoprotein (S glycoprotein) attaches the virion to the host cell membrane and plays a major role in utilizing angiotensin converting enzyme 2 as a receptor [16]. 2. Another proposed mechanism is the use of cell-surface heparan sulfate proteoglycans (by SARS-CoV). This mechanism is probably responsible for the coagulopathy manifested in patients lungs and leading to respiratory failure [17]. 3. A third mechanism involves SARS-CoV 2 Attacking the 1-beta chain of hemoglobin bringing about the release of iron with its multitude of toxic effects[18].

Here, we worked to explore the potential for existing natural or synthetic drugs to take down the new virus SARS-CoV-2. Recently, a molecular docking study was reported, which identifies small-molecules that bind to either the isolated Viral S-protein at its host receptor region or to the S protein-human ACE2 interface using the world's most powerful supercomputer, SUMMIT [19]. Electronic characteristics were not identified or discussed. Through our work reported here, we are exploring in a simplified way binding capability of drugs/nutraceuticals to accelerate the discovery of improved treatment options thus paving the way to a hopefully near future eradication of the COVID-19 pandemic. The results presented are a first step towards the identification of the importance of the electronic characteristics of nutraceuticals and exploitation in treatments with 
potentially tolerable side-effects relative to the current scarce antiviral drugs against COVID-19. In this study, we focused on the interaction of different molecules with the spike glycoprotein of SARS-CoV 2. We also tested the interaction of the spike glycoprotein with hemoglobin and heparin even though this might not be the main mechanism [20]. In-vitro and in-vivo validation of the simulated results is of urgent demand.

\section{2- Simulation Methodology}

Equilibrium geometry of the interacting molecules are obtained by applying molecular mechanics method built in Spartan 18 parallel suite (core > 16) package offered by www. Wavefun.com at reduced price due to COVID-19 lockdown. Single point computations are then applied to the molecular mechanically optimized equilibrium geometry with DFT using the well-known B3LYP/6$31 \mathrm{G}(\mathrm{d})$ method. This method is suitable and cost effective. Other more expensive functional such as WB97X-D or higher bases sets did not result in any significant change in the results obtained.

\section{3- Results and Discussion}

We selected the drugs with considerable binding energy with S protein and/or ACE2 (Table 1). We selected the sequence LOOP1 (receptor binding loop)-LYS-ARG-GLY-VAL [21] to represent coronavirus S-protein function that mediates receptor binding and fusion of the viral and host cell membrane. HIS-GLU-GLY-PRO-LEU-HIS-LYS protein sequence has been reasonably assumed to represent ACE2 enzyme [22] .

Guided by electrostatic potential energy maps (ESP-maps) that characterize the electron distribution surface of molecules and the energies of HOMO and LUMO of the interacting species, manual docking is successfully performed. The binding energy of a drug and S protein or ACE2 is calculated using the well-known equation

$B E_{\text {adduct }}=E_{\text {adduct }}-\left(E_{\text {drug }}+E_{\text {peptide }}\right)$

Negative value of $B E$ indicates formation of stable adduct. The results are summarized in Tables 1, 2 and 3. Table 1 shows the frontier orbital (HOMO-LUMO) energy values that reflect the electronic donor-acceptor reactivity of the individual molecules. Stability of the adducts given in Table 2 and 3 originates from hydrogen bond formation and other electrostatic attractive forces due to HOMO - LUMO electron transfer capabilities between the interacting molecules. Figures 
1-8 depicts the shapes and the HOMO (mesh format) - UMO (transparent) interactions between the constituents of some representative adducts.

Table 1- HOMO - LUMO (eV) of individual molecules

\begin{tabular}{|l|c|c|}
\hline Drug or species & $\mathrm{HO}$ & $\mathrm{LU}$ \\
\hline Hydroxychloroquine & -5.76 & -1.73 \\
\hline Raspberry-ketone & -5.84 & -0.47 \\
\hline Ivermectin & -5.74 & -0.79 \\
\hline Menthol & -6.89 & 2.16 \\
\hline Eriodictyol & -5.76 & -1.5 \\
\hline Thymoquinone & -7.11 & -3.23 \\
\hline Resveratrol & -5.29 & -1.27 \\
\hline Favipiravir & -6.86 & -2.1 \\
\hline Quercetin & -5.48 & -1.87 \\
\hline Curcumin & -5.7 & -2.21 \\
\hline Nicolosamide & -6.39 & -2.76 \\
\hline S protein (Beta Sheet) & -5.27 & -0.29 \\
\hline ACE2 & -5.97 & -0.93 \\
\hline Heparin & -6.97 & -0.52 \\
\hline Heme & -4.75 & -2.23 \\
\hline
\end{tabular}

Table 2- HOMO - LUMO energies (eV) and Binding energy, BE of the adducts: S-protein - Drug and S Protein ACE2, Hemoglobin and Heparin

\begin{tabular}{|l|c|c|l|}
\hline Drug/receptor: S Protein Adduct & HO & LU & BE/Kcal/mol \\
\hline Hydroxychloroquine & -5.76 & -1.73 & -16.4 \\
\hline Raspberry-ketone & -5.28 & -0.39 & -15.51 \\
\hline Ivermectin & -5.3 & -0.74 & -14.13 \\
\hline Menthol & -5.27 & -0.3 & -13.39 \\
\hline Eriodictyol & -5.29 & -1.34 & -11.36 \\
\hline Thymoquinone & -5.28 & -3.18 & -9.54 \\
\hline Resveratrol & -5.3 & -1 & -8.23 \\
\hline Favipiravir & -5.34 & -2.35 & -7.55 \\
\hline Quercetin & -5.23 & -1.62 & -3.64 \\
\hline Curcumin & -5.25 & -1.98 & $+\mathrm{ve}$ \\
\hline Nicolosamide & -5.26 & -3.47 & $+\mathrm{ve}$ \\
\hline ACE2 & -5.35 & -0.91 & -14.54 \\
\hline Heme & -4.28 & -2.20 & $+\mathrm{ve}$ \\
\hline Heparin & -5.37 & -0.77 & $+\mathrm{ve}$ \\
\hline
\end{tabular}




\section{Table 3- HOMO - LUMO energies (eV) and Binding energy, BE of the adducts: ACE2 - Drug}

\begin{tabular}{|c|c|c|c|}
\hline Drug: ACE2 & $\mathrm{HO}$ & $\mathrm{LU}$ & $\mathrm{BE} / \mathrm{Kcal} / \mathrm{mol}$ \\
\hline Favipiravir & -5.90 & -2.40 & -11.03 \\
\hline Nicolosamide & -6.01 & -3.13 & -9.94 \\
\hline Hydroxychloroquine & -5.66 & -1.44 & -7.83 \\
\hline Eriodictyol & -5.77 & -1.34 & -2.26 \\
\hline Menthol & -5.98 & -0.93 & -1.75 \\
\hline Thymoquinone & -5.96 & -3.25 & -1.38 \\
\hline Raspberry-ketone & -5.71 & -1.00 & $+\mathrm{ve}$ \\
\hline Resveratrol & -5.54 & -0.95 & $+\mathrm{ve}$ \\
\hline Quercetin & -5.64 & -1.73 & $+\mathrm{ve}$ \\
\hline Curcumin & -5.67 & -2.07 & $+\mathrm{ve}$ \\
\hline
\end{tabular}

11 ligands (7 natural and 4 synthesized antivirals) were calculated to bind to either the Sprotein(Figure 1): ACE2 (Figure 1) interface (Figure 2) - ligand binding complex (Figure 3 as an example of the case of favipiravir) or the binding-interface of the isolated S-protein, ACE2, Heme (Figure 7) or Heparin (Figure 8) [18, 20]. We assume the identified drugs could be positioned to limit viral recognition of host cells or disturb host-virus. Hydroxychloroquine, ivermectin and favipiravir are strongest binding drugs to both $\mathrm{S}$ protein and ACE2, while raspberry-ketone, menthol, eriodictyol and thymoquinone are potentially bound to the viral S protein (Table 2). Raspberry-ketone is of comparable binding strength to the known antiviral drugs investigated. Moreover, it seems to block or at least disturb S protein - ACE2 interactions. Other natural drugs did not show affinity to bind to either S protein or ACE2. However, they disturb direct S protein - ACE2 interaction by showing capabilities to act as electron sink by receiving electrons into its disturbed low-laying LUMO from the strong nucleophile S protein in presence of ACE2. This is attributed to the low-laying LUMO of most of the drugs relative to that of ACE2 (see Figure 3 as an example).

Finally, no noticeable stable adducts due to interactions between S protein and hemoglobin or heparin was noticed (See Table 2 and Figures 7 and 8). However, only thymoquinone showed capability to interact with hemoglobin as electron sink due to its low-laying LUMO orbital. 


\section{4- Conclusions}

11 ligands (7 natural and 4 synthesized antivirals) were calculated to bind to either the S-protein: ACE2 interface-ligand binding complex or the binding-interface of the isolated S-protein, ACE2, Heme or Heparin. We showed that the identified drugs could be positioned to limit viral recognition of host cells or disturb host-virus. Simplified assumptions of Frontier orbitals interactions are successful in explaining the results. Generally, in presence of ACE2 enzyme, drugs act as Lewis acids (or electron sink molecules) towards the viral S protein that exhibits strong electrophilic property. Hydroxychloroquine, ivermectin and favipiravir are strongest binding drugs to both S protein and ACE2, while raspberry-ketone, menthol, eriodictyol and thymoquinone are potentially bind to $\mathrm{S}$ protein. Raspberry-ketone is of comparable binding strength to hydroxychloroquine and ivermectin antiviral drugs, which are currently clinically investigated. Moreover, raspberry ketone seems to block or at least disturb S protein - ACE2 interactions. Other natural drugs did not show affinity to bind to either S protein or ACE2. However, they disturb direct S protein - ACE2 interaction by showing capabilities to receive electrons (electron sink) into its disturbed LUMO from $\mathrm{S}$ protein in presence of ACE2. Thymoquinone is the only drug among the tested ones that showed electron acceptor capability towards heme.

\section{Conflict of interests}

There are no conflicts of interest to declare. All data are available to share upon request from the corresponding author. 


\section{Figures}
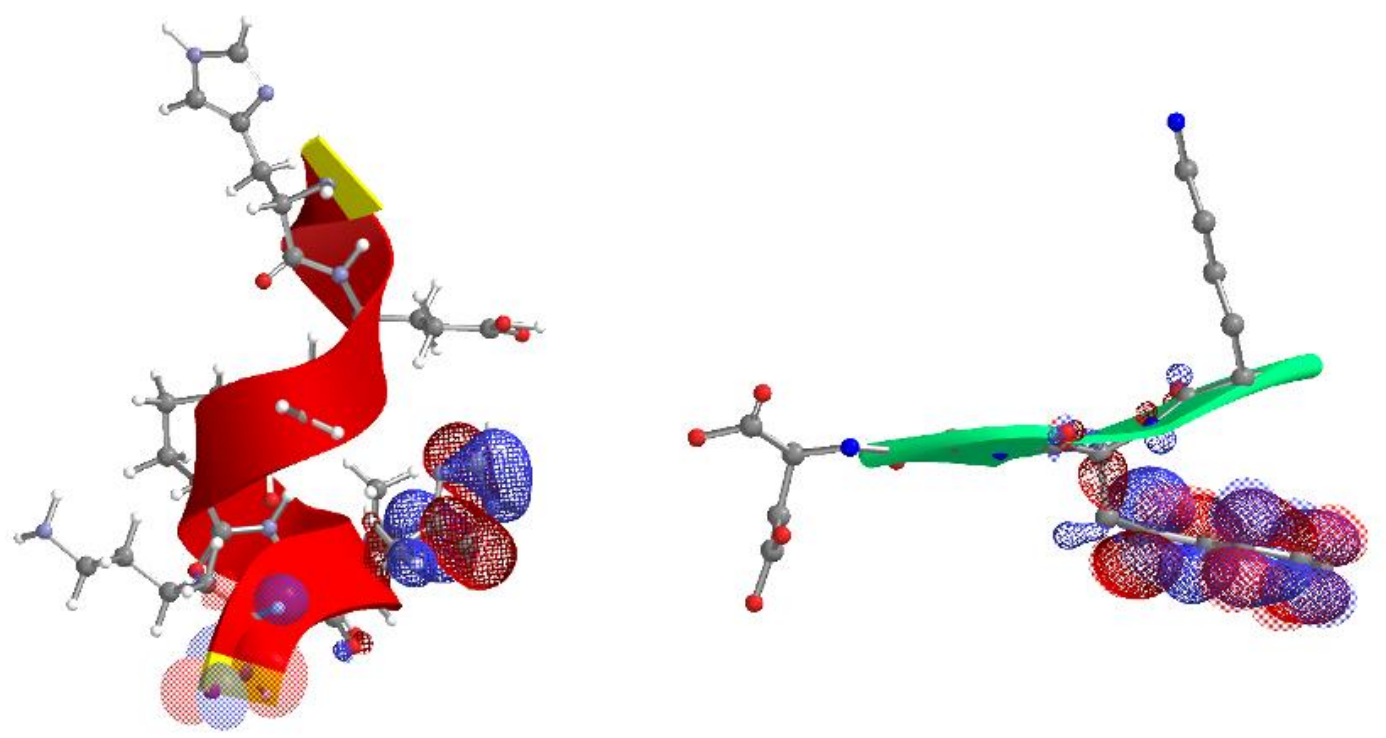

Figure 1. ACE2 protein sequence (alpha helix) and HOMO (mesh) - LUMO (transparent)

(left pane) and S-protein Beta-sheet sequence (right pane)

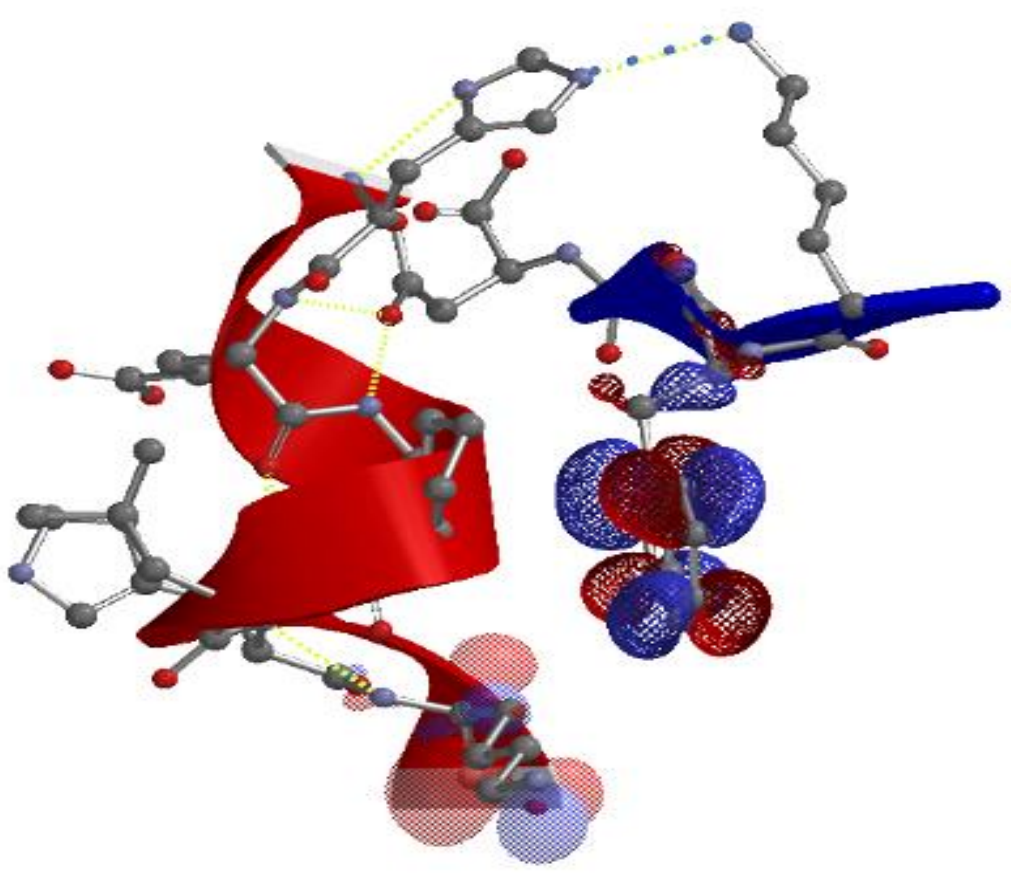

Figure 2. ACE2 - S protein complex. Dots (blue) represent hydrogen bond formation between the interacting species. HOMO (mesh) localised on the S protein sheet (representing its nucleophilic character) and LUMO (transparent) on ACE2 enzyme. 


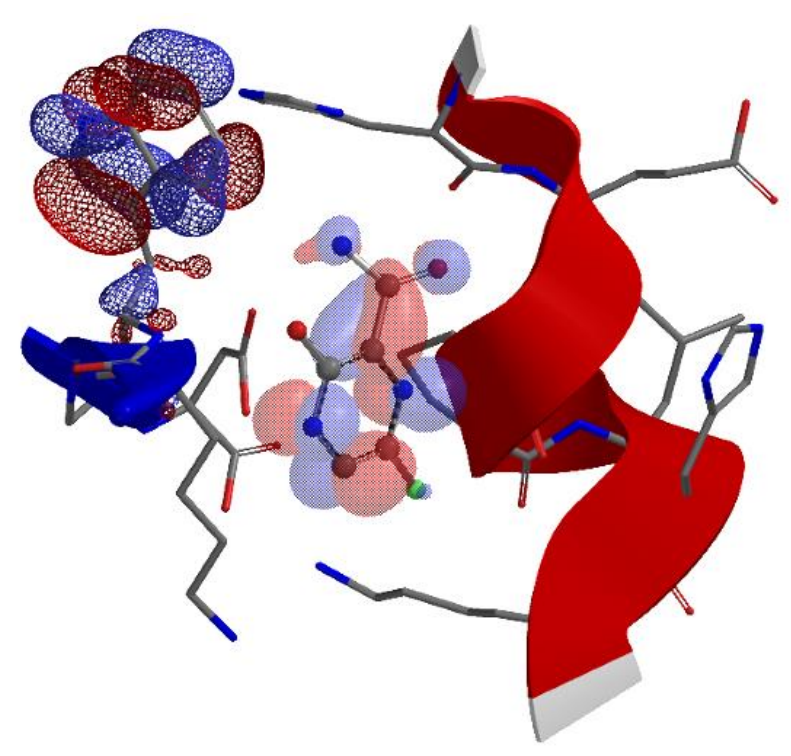

Figure 3. Favipiravir drug positioned between S protein and ACE2 enzyme, which inhibits direct interaction between them and acts as electron sink of the $\mathrm{S}$ protein nucleophile.
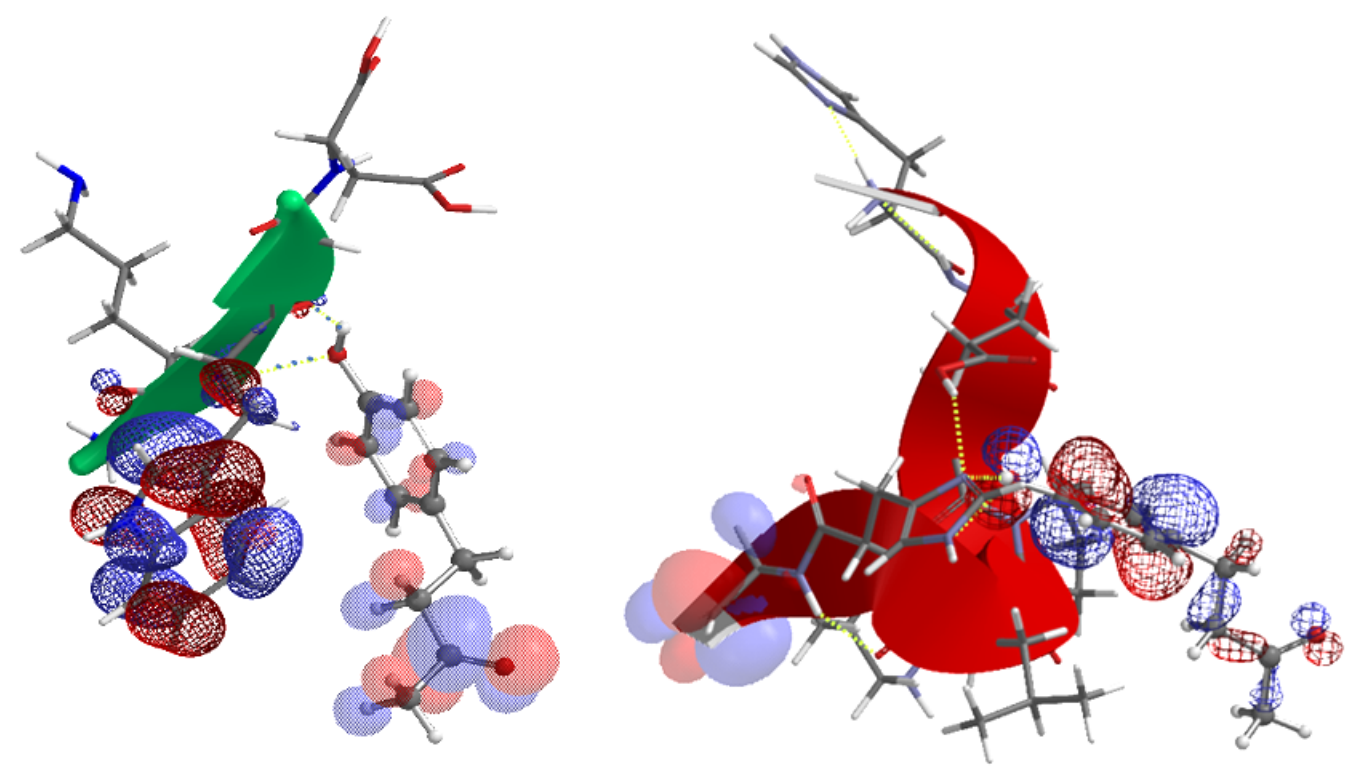

Figure 4. S protein sheet - Raspberry-ketone HOMO - LUMO (nucleophilic - electrophilic) interactions and Hydrogen bond formation (Left pane). Right pane shows ACE2 enzyme - Raspberry-ketone adduct where Raspberry-ketone reacts as a nucleophile to the ACE2 electrophile. 


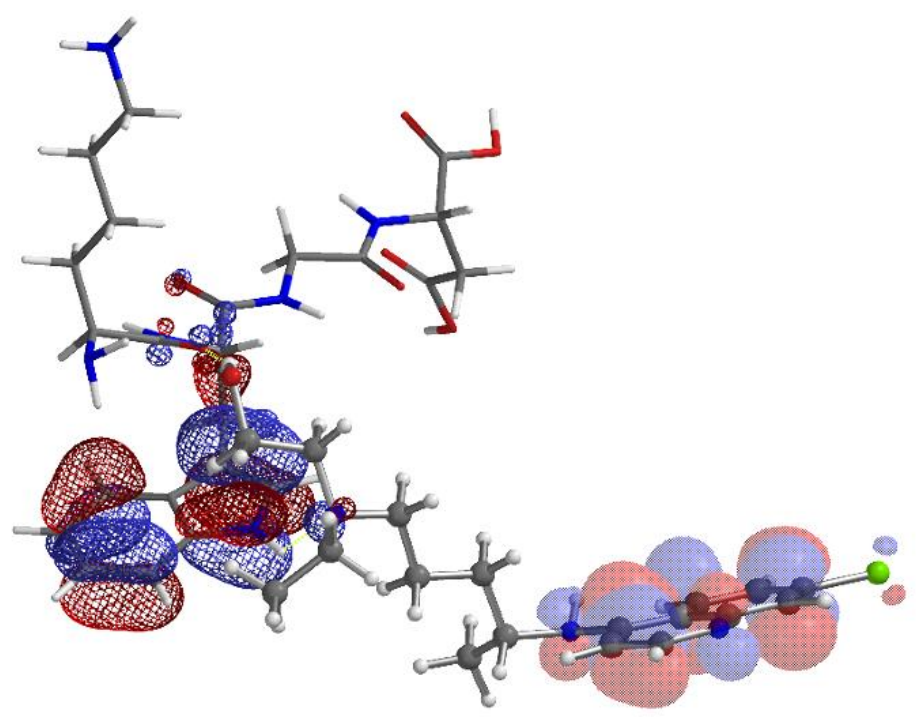

Figure 5. Hydroxychloroquine drug - $\mathrm{S}$ protein sheet (Tube) stable adduct. Hydroxychloroquine acts as electron sink (LUMO is localized on the drug).

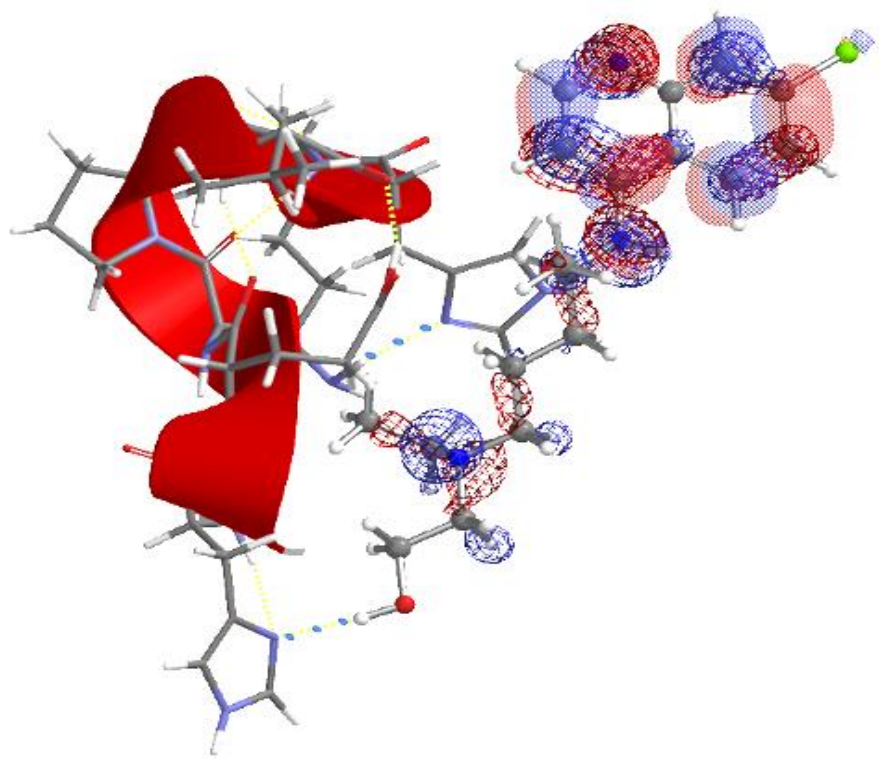

Figure 6. ACE2 - Hydroxychloroquine drug adduct with only hydrogen-bonds formation. Both HOMO-LUMO are localised on the drug molecule. 

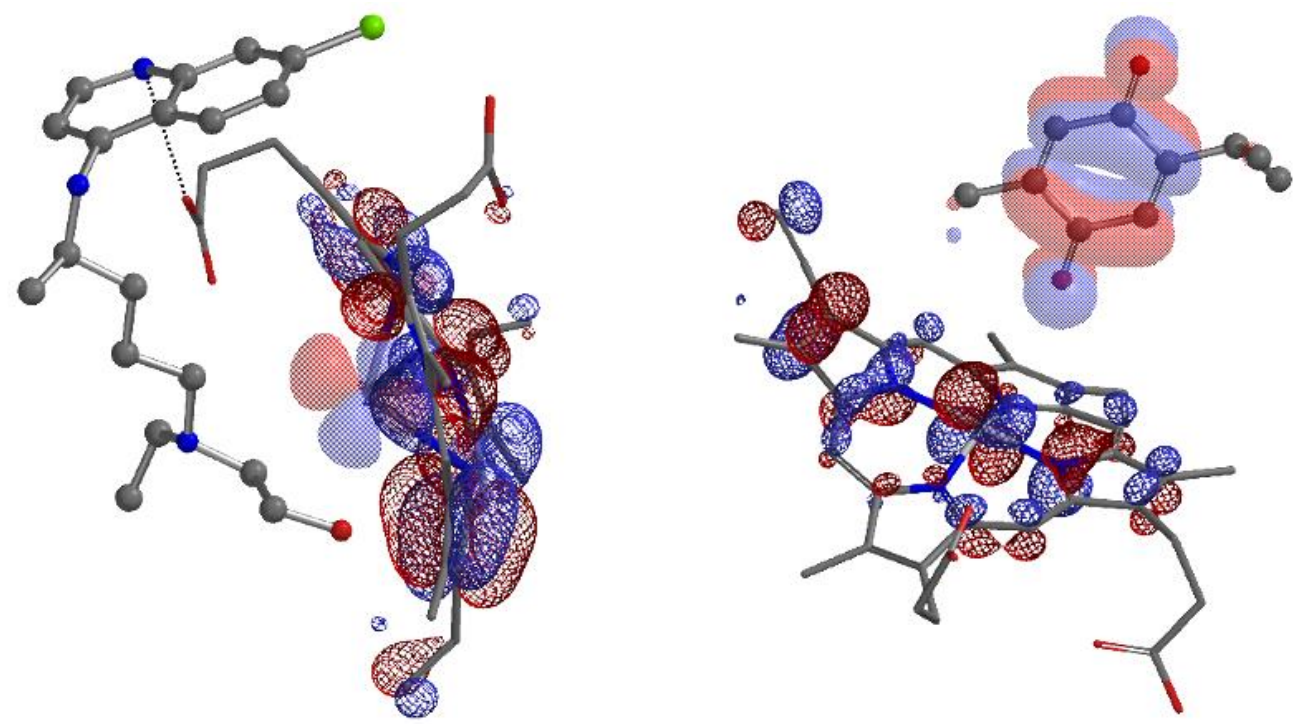

Figure 7. Haemoglobin - Hydroxychloroquine interaction (Left pane) showing Hydrogen bond formation without HOMO-LUMO interaction. Right pane shows thymoquinone, which acts as electron sink instead of the Fe ion on the haemoglobin. S protein behaves similarly to Hydroxychloroquine towards haemoglobin where (HOMO-LUMO) are of heme character.

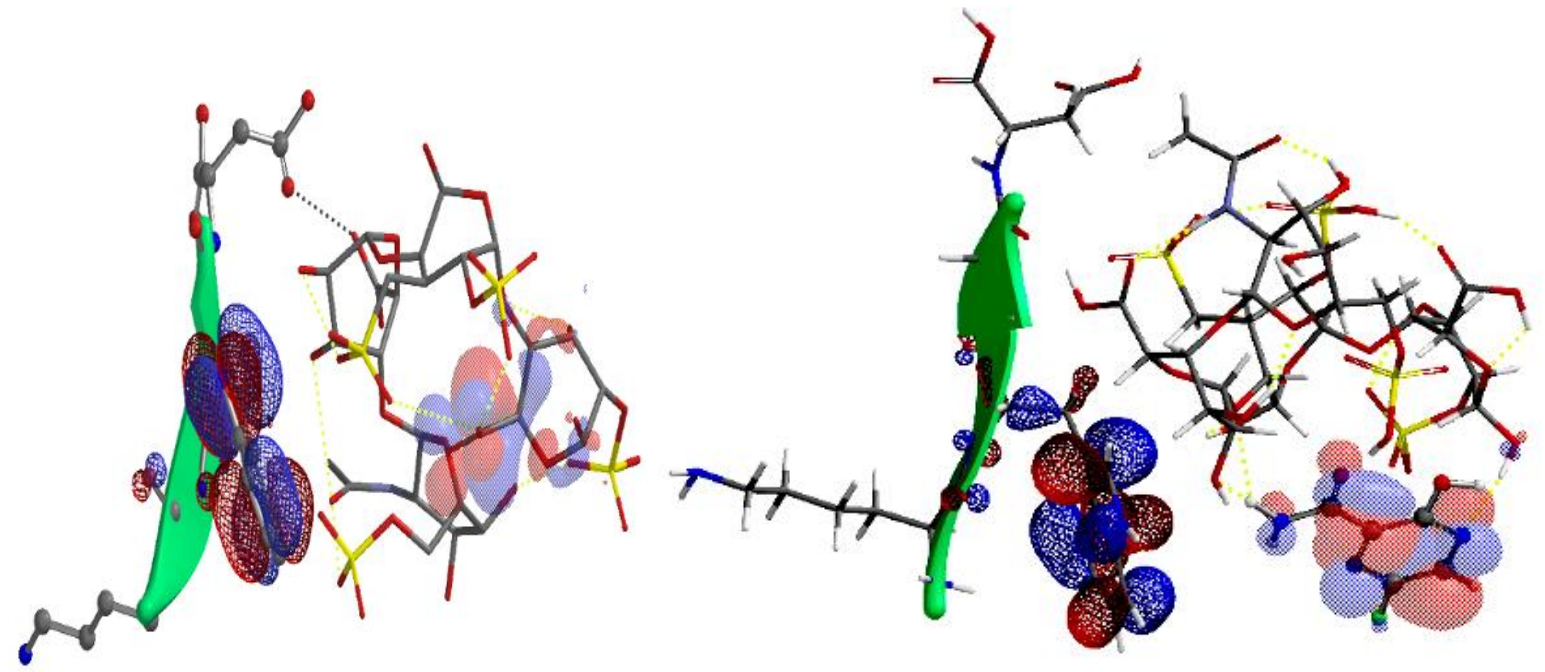

Figure 8. Left side: S protein (HOMO) - heparin (LUMO) interaction and hydrogen bond formation (Black dots). Right side: Favipiravir acts as electron sink and disrupt S protein - heparin direct interaction. 


\section{References}

[1] M. Costanzo, M.A.R. De Giglio, G.N. Roviello, SARS CoV-2: Recent Reports on Antiviral Therapies Based on Lopinavir/Ritonavir, Darunavir/Umifenovir, Hydroxychloroquine, Remdesivir, Favipiravir and Other Drugs for the Treatment of the New Coronavirus, Curr Med Chem (2020). [2] C.K. Lumby, L. Zhao, M. Oporto, T. Best, H. Tutill, D. Shah, P. Veys, R. Williams, A. Worth, C.J.R. Illingworth, J. Breuer, Favipiravir and Zanamivir Cleared Infection with Influenza B in a Severely Immunocompromised Child, Clin Infect Dis (2020).

[3] L. Caly, J.D. Druce, M.G. Catton, D.A. Jans, K.M. Wagstaff, The FDA-approved Drug Ivermectin inhibits the replication of SARS-CoV-2 in vitro, Antiviral Res (2020) 104787.

[4] J. Xu, P.Y. Shi, H. Li, J. Zhou, Broad Spectrum Antiviral Agent Niclosamide and Its Therapeutic Potential, ACS Infect Dis (2020).

[5] Y.S. Abulfadl, N.N. El-Maraghy, A.E. Ahmed, S. Nofal, Y. Abdel-Mottaleb, O.A. Badary, Thymoquinone alleviates the experimentally induced Alzheimer's disease inflammation by modulation of TLRs signaling, Hum Exp Toxicol 37(10) (2018) 1092-1104.

[6] Y. Atef, H.M. El-Fayoumi, Y. Abdel-Mottaleb, M.F. Mahmoud, Quercetin and tin protoporphyrin attenuate hepatic ischemia reperfusion injury: role of HO-1, Naunyn Schmiedebergs Arch Pharmacol 390(9) (2017) 871-881.

[7] R.T. Attia, Y. Abdel-Mottaleb, D.M. Abdallah, H.S. El-Abhar, N.N. El-Maraghy, Raspberry ketone and Garcinia Cambogia rebalanced disrupted insulin resistance and leptin signaling in rats fed high fat fructose diet, Biomed Pharmacother 110 (2019) 500-509.

[8] Y.A.M. Nada Ismail, Amani Ali Eissa Ahmed, Nabila N El-Maraghy, Novel combination of thymoquinone and resveratrol enhances anticancer effect on hepatocellular carcinoma cell line, Future Journal of Pharmaceutical Sciences 1(4) (2018) 41-46.

[9] D. Barreca, G. Gattuso, E. Bellocco, A. Calderaro, D. Trombetta, A. Smeriglio, G. Lagana, M. Daglia, S. Meneghini, S.M. Nabavi, Flavanones: Citrus phytochemical with health-promoting properties, Biofactors 43(4) (2017) 495-506.

[10] A.A. Bertelli, C. Mannari, S. Santi, C. Filippi, M. Migliori, L. Giovannini, Immunomodulatory activity of shikimic acid and quercitin in comparison with oseltamivir (Tamiflu) in an in vitro model, J Med Virol 80(4) (2008) 741-5.

[11] A. Capci Karagoz, C. Reiter, E.J. Seo, L. Gruber, F. Hahn, M. Leidenberger, V. Klein, F. Hampel, O. Friedrich, M. Marschall, B. Kappes, T. Efferth, S.B. Tsogoeva, Access to new highly potent antileukemia, antiviral and antimalarial agents via hybridization of natural products (homo)egonol, thymoquinone and artemisinin, Bioorg Med Chem 26(12) (2018) 3610-3618.

[12] J.H. Lee, M. Oh, J.H. Seok, S. Kim, D.B. Lee, G. Bae, H.I. Bae, S.Y. Bae, Y.M. Hong, S.O. Kwon, D.H. Lee, C.S. Song, J.Y. Mun, M.S. Chung, K.H. Kim, Antiviral Effects of Black Raspberry (Rubus coreanus) Seed and Its Gallic Acid against Influenza Virus Infection, Viruses 8(6) (2016).

[13] S.C. Lin, C.T. Ho, W.H. Chuo, S. Li, T.T. Wang, C.C. Lin, Effective inhibition of MERS-CoV infection by resveratrol, BMC Infect Dis 17(1) (2017) 144. 
[14] S.Z. Moghadamtousi, H.A. Kadir, P. Hassandarvish, H. Tajik, S. Abubakar, K. Zandi, A review on antibacterial, antiviral, and antifungal activity of curcumin, Biomed Res Int 2014 (2014) 186864.

[15] D.J.R. Taylor, S.M. Hamid, A.M. Andres, H. Saadaeijahromi, H. Piplani, J.F. Germano, Y. Song, S. Sawaged, R. Feuer, S.J. Pandol, J. Sin, Antiviral Effects of Menthol on Coxsackievirus B, Viruses 12(4) (2020).

[16] P. Habibzadeh, E.K. Stoneman, The Novel Coronavirus: A Bird's Eye View, Int J Occup Environ Med 11(2) (2020) 65-71.

[17] J. Lang, N. Yang, J. Deng, K. Liu, P. Yang, G. Zhang, C. Jiang, Inhibition of SARS pseudovirus cell entry by lactoferrin binding to heparan sulfate proteoglycans, PLoS One 6(8) (2011) e23710.

[18] L. Wenzhong, L. Hualan, COVID-19: Attacks the 1-Beta Chain of Hemoglobin and Captures the Porphyrin to Inhibit Human Heme Metabolism, ChemRxiv. (2020).

[19] M.S. Smith, Jeremy C. , Repurposing Therapeutics for COVID-19:

Supercomputer-Based Docking to the SARS-CoV-2 Viral Spike Protein and Viral Spike ProteinHuman ACE2 Interface. , ChemRxiv (2020).

[20] Courtney Mycroft-West, Dunhao Su, Stefano Elli, Scott Guimond, Gavin Miller, Jeremy Turnbull, Edwin Yates, Marco Guerrini, David Fernig, Marcelo Lima, M. Skidmore, The 2019 coronavirus (SARS-CoV-2) surface protein (Spike) S1 Receptor Binding Domain undergoes conformational change upon heparin binding, BioRxiv (2020).

[21] Z. Li, A.C. Tomlinson, A.H. Wong, D. Zhou, M. Desforges, P.J. Talbot, S. Benlekbir, J.L. Rubinstein, J.M. Rini, The human coronavirus HCoV-229E S-protein structure and receptor binding, Elife 8 (2019).

[22] W. Li, M.J. Moore, N. Vasilieva, J. Sui, S.K. Wong, M.A. Berne, M. Somasundaran, J.L. Sullivan, K. Luzuriaga, T.C. Greenough, H. Choe, M. Farzan, Angiotensin-converting enzyme 2 is a functional receptor for the SARS coronavirus, Nature 426(6965) (2003) 450-4. 